\title{
A COMPLETE LOCAL FACTORIAL RING OF DIMENSION 4 WHICH IS NOT COHEN-MACAULAY
}

\author{
BY ROBERT M. FOSSUM AND PHILLIP A. GRIFFITH ${ }^{1}$
}

Communicated by Hyman Bass, July 15, 1974

Samuel [7] stated that he knew of no factorial noetherian ring which was not Cohen-Macaulay. Murthy [6] showed that a geometric factorial ring which is Cohen-Macaulay is Gorenstein. Subsequently, Bertin [1] constructed an example of a factorial ring which was not Cohen-Macaulay. Hochster and Roberts [5] noticed that such examples abound and were found by Serre [9]. On the other hand, Raynaud, Boutot, and Hartshorne and Ogus [3] have shown that a complete local ring which is factorial, of dimension at most 4 , and with C as residue class field is Cohen-Macaulay.

This note is to announce that the completion of Bertin's example (which is characteristic 2) is factorial. This defeats a conjecture suggested by Example 5.9 of Hochster [4] which states: If $A$ is a complete noetherian domain, then some symbolic power of a prime ideal of height one is a maximal CohenMacaulay module.

Let $k$ be a perfect field of characteristic $p$ with $p \neq 0$. Let $N$ operate on $k^{4}$ by $N\left(e_{i}\right)=e_{i+1}$ for $1 \leqslant i<4$ and with $N\left(e_{4}\right)=0$. Then $I+N$ is an automorphism of $k^{4}$ of order $p$ if $p \geqslant 5$ and of order 4 if $p=2$. Let $B=k\left[X_{1}, X_{2}, X_{3}, X_{4}\right]$, which we consider to be the symmetric algebra on $k^{4}$. Let $G$ denote the group of automorphisms of $B$ induced by $I+N$. It follows from Samuel [8] that the ring of invariants $A=B^{G}$ is factorial. If $p=2$, then Bertin [1] has shown that $A$ is not CohenMacaulay. Using a result in Serre [9], Hochster and Roberts [5] show that $A$ is not Cohen-Macaulay if $p \geqslant 5$. Let $S=B_{m}$ and let $R=S^{G}$, where $m=\left(X_{1}, X_{2}, X_{3}, X_{4}\right)$. It follows that $R=A_{n}$ with $n=m \cap A$. The different $D(S / R)=S$, and therefore the cohomology group $H^{1}\left(G, \mathbf{G}_{m}(S)\right)$ $=0$. Let $\hat{S}$ denote the $m$-adic completion of $B$. The first result is almost obvious.

AMS (MOS) subject classifications (1970). Primary 13F15, $13 \mathrm{H} 10$.

${ }^{1}$ This research was partially supported by the NSF. 
PROPOSITION 1. The n-adic completion of $R$ is the ring of G-invariants of $\hat{S}$. That is $\hat{R}=\hat{S}^{G}$.

This yields the following corollary.

COROLlary 2. The ring $\hat{R}$ is not Cohen-Macaulay.

Let $U_{n}=1+m^{n} S$ and $\hat{U}_{n}=1+m^{n} \hat{S}$. The $U_{n}$ are subgroups of $\mathbf{G}_{m}(S)$, and the following sequences are exact as $G$-modules:

$$
\begin{gathered}
1 \rightarrow U_{1} \rightarrow \mathbf{G}_{m}(S) \rightarrow k^{*} \rightarrow 1, \\
1 \rightarrow U_{n+1} \rightarrow U_{n} \rightarrow m^{n} / m^{n+1} \rightarrow 0
\end{gathered}
$$

(and similarly with hats everywhere). Since $G$ is cyclic, the cohomology of $G$ is periodic of period 2 (cf. Cartan and Eilenberg [2]). We will study the exact sequence

$$
\begin{aligned}
\cdots \rightarrow \hat{H}^{0}\left(G, m^{n} / m^{n+1}\right) & \rightarrow H^{1}\left(G, U_{n+1}\right) \rightarrow H^{1}\left(G, U_{n}\right) \\
& \rightarrow H^{1}\left(G, m^{n} / m^{n+1}\right) \rightarrow H^{2}\left(G, U_{n+1}\right) \\
& \rightarrow H^{2}\left(G, U_{n}\right) \rightarrow H^{2}\left(G, m^{n} / m^{n+1}\right) \rightarrow \cdots
\end{aligned}
$$

and the corresponding one with hats. Note that the $G$-module $m^{n} / m^{n+1}$ is the $n$th symmetric power of $m / m^{2}$ as a $G$-module.

Proposition 3. The connecting homomorphisms $\hat{H}^{0}\left(G, m^{n} / m^{n+1}\right)$ $\rightarrow H^{1}\left(G, U_{n+1}\right)$ are zero for all $n$. Therefore the groups $H^{1}\left(G, U_{n}\right)$ are zero and the sequence

$$
\begin{aligned}
0 & \rightarrow H^{1}\left(G, \hat{U}_{n+1}\right) \rightarrow H^{1}\left(G, \hat{U}_{n}\right) \rightarrow \cdots \\
& \rightarrow H^{2}\left(G, \hat{U}_{n}\right) \rightarrow H^{2}\left(G, m^{n} / m^{n+1}\right) \rightarrow 0
\end{aligned}
$$

is exact.

REMARK. The contragredient representation of $G$ on the $k$-duals of $m^{n} / m^{n+1}$ induces isomorphisms of $k$-vector spaces:

$$
H^{1}\left(G, m^{n} / m^{n+1}\right)=H^{2}\left(G,\left(m^{n} / m^{n+1}\right)^{\swarrow}\right)^{\swarrow} .
$$

To show that $\hat{R}$ is factorial, it is sufficient, therefore, to show that the homomorphisms $H^{1}\left(G, \hat{U}_{n}\right) \rightarrow H^{1}\left(G, m^{n} / m^{n+1}\right)$ are zero for all $n$. In characteristic $p=2$, this is accomplished by directly calculating the groups $H^{1}\left(G, m^{n} / m^{n+1}\right)$ and then showing that the connecting homomorphisms to $H^{2}\left(G, U_{n+1}\right)$ are injections. Similar arguments should suffice in characteristic $p \geqslant 5$. 
Proposition 4. Suppose char $k=2$. If $n$ is odd, then $H^{1}\left(G, m^{n} / m^{n+1}\right)=0$. If $n$ is even, then $\operatorname{dim}_{k} H^{1}\left(G, m^{n} / m^{n+1}\right)=[n / 4]+$ 1. If $n=4 k$ and $x=X_{1}\left(X_{1}+X_{3}\right)$ (which is just $X_{1} \cdot(I+N)^{2}\left(X_{1}\right)$ ), then a basis for $H^{1}\left(G, m^{n} / m^{n+1}\right)$ is given by the classes of $x^{2 k}, x^{2 k-1} a(x)$, $\cdots, x^{k} a(x)^{k}$. A basis for $H^{2}\left(G, m^{n} / m^{n+1}\right)$ is given by the classes of $(x+a(x))^{2 k}, x^{2 k-1} a(x)+x a(x)^{2 k-1}, \cdots, x^{k} a(x)^{k}$, where $a(x)=$ : $(I+N)(x)$ (and similarly for $n=4 k+2)$.

The results announced here, as well as similar ones for $\mathbf{Z} / p \mathbf{Z}$ acting on $k\left[\left[X_{0}, \cdots, X_{p-1}\right]\right]$, will appear elsewhere.

\section{REFERENCES}

1. M.-J. Bertin, Anneaux d'invariants d'anneaux de polynomes, in caracteristique p, C. R. Acad. Sci. Paris 264 (1967), 653-656.

2. H. Cartan and S. Eilenberg, Homological algebra, Princeton Univ. Press, Princeton, N. J., 1956. MR 17, 1040.

3. R. Hartshorne and A. Ogus, On the factoriality of local rings of small embedding codimension Communications in Algebra 1 (1974), 415-437.

4. M. Hochster, Cohen-Macaulay modules, Conf. on Commutative Algebra, Lecture Notes in Math., vol. 311, Springer-Verlag, Berlin and New York, 1973, pp. 120-152.

5. M. Hochster and J. Roberts, Rings of invariants of reductive groups acting on regular rings are Cohen-Macaulay. Advances in Math. 13 (1974), 115-175.

6. M. P. Murthy, A note on factorial rings, Arch. Math. 15 (1964), 418-420. MR $30 \# 3905$.

7. P. Samuel, On unique factorization domains, Illinois J. Math. 5 (1961), 1-17. MR 22 \#12121.

8. - Classes de diviseurs et dérivées logarithmiques, Topology 3 (1964), suppl. 1, 81-96. MR 29 \#3490.

9. J.-P. Serre, Sur la topologie des variétés algébriques en caractéristique p, Internat. Sympos. on Algebraic Topology, Universidad Nacional Autónoma de México and UNESCO, México City, 1958, pp. 24-53. MR 20 \#4559.

DEPARTMENT OF MATHEMATICS, UNIVERSITY OF ILLINOIS AT URBANACHAMPAIGN, URBANA, ILLINOIS 61801 